\section{Extensión en Colabor: producción de prácticas autogestionadas de formación para la gestión colectiva del trabajo}

\author{
Sebastián Carenzo \\ CONICET - Instituto de Estudios sobre la \\ Ciencia y la Tecnología, Universidad Nacional \\ de Quilmes, Argentina. \\ sebastian.carenzo@gmail.com \\ (iD) orcid.org/0000-0002-6870-6937 \\ María Inés Fernández Álvarez \\ CONICET - Instituto de Ciencias \\ Antropológicas, Universidad de Buenos Aires, \\ Argentina
}

RECEPCIÓN: 24/06/19

ACEPTACIÓN FINAL: 19/11/19

\section{Resumen}

Este artículo comparte los resultados de un proyecto de extensión universitaria cuyo principal objetivo consistió en desarrollar contenidos y metodologías destinados a fortalecer prácticas autogestionadas de formación para la gestión colectiva del trabajo a partir de una dinámica colaborativa entre trabajadoras/es de universidades y cooperativas. La reflexión que proponemos se focaliza en la productividad cultural de estas iniciativas para disputar prácticas y sentidos en torno a los procesos de formación y capacitación para el trabajo implementados en estas experiencias. El proyecto se materializó en la elaboración de la Plataforma Co-labor, una interfase en línea de acceso abierto, libre e irrestricto, a contenidos y propuestas pedagógicas elaborados sobre la base de la sistematización de los aprendizajes generados desde y para estas experiencias de gestión colectiva del trabajo Así, a través de la reflexión sobre esta iniciativa buscamos deliberadamente abordar la cuestión formativa en espacios laborales recuperados y/o conquistados mediante luchas populares, siempre tensionados por dinámicas de desterritorialización-reterritorialización de sus prácticas económicas, sociales y culturales. En esta tensión situamos la reflexión sobre los aprendizajes derivados del trabajo del equipo con las cooperativas 19 de Diciembre y Textiles Pigué en torno a los aportes de la experiencia colaborativa para repensar modalidades de extensión universitaria.

Palabras clave: formación, plataforma digital, cooperativas, trabajo, diseño colaborativo. mifernandezalvarez@gmail.com
(D) orcid.org/0000-0003-4201-2694

Sapienza Università di Roma, Italia. Instituto de Altos Estudios Sociales, Universidad Nacional de San Martín, Argentina ali.castronovo@gmail.com

\section{Alioscia Castronovo}

(iD) orcid.org/0000-0002-8339-4791
Collaborative university extension activities: the production of self-managed training practices for collective management of work

\section{Abstract}

This article seeks to share the results of a university extension project whose main objective was to develop contents and methodologies with the purpose of strengthening self-managed training practices for collective work management based on a collaborative dynamic between university workers and cooperatives. The reflection that we propose focuses on the cultural productivity of these initiatives to dispute practices and senses over the processes of training and training for work implemented in these experiences. The project materialized in the elaboration of the Co-labor Platform, an open access online interface that guarantees open and unrestricted access to contents and pedagogical proposals elaborated from the systematization of the learning generated from and for these management experiences collective work. Thus, through reflecting on this initiative we seek to address the formative issue in work spaces recovered and / or conquered through popular struggles, always stressed by the dynamics of deterritorialization-reterritorialization of their economic, social and cultural practices. In this tension we place the reflection on the learning derived from the work of the team with the cooperatives December 19 and Textiles I continued reflecting on the contributions of this collaborative experience to rethink modalities of university extension.

Keywords: education, digital platform, labour cooperatives, collaborative design.
Cultura(s) en clave de extensión universitaria / Intervenciones 다(1)(2)

\section{Elisa Gigliarelli}

Programa Facultad Abierta de la Facultad de Filosofía y Letras, Universidad de Buenos Aires, Argentina. elisa.officine@gmail.com (iD orcid.org/0000-0001-9003-4877
Extensão em Colabor: a produção de práticas de treinamento autogerenciado para gestão coletiva do trabalho

\section{Resumo}

Este artigo busca compartilhar os resultados de um projeto de extensão universitária, cujo objetivo principal foi o desenvolvimento de conteúdos e metodologias voltadas ao fortalecimento de práticas de treinamento autogerenciado em gestão coletiva do trabalho com base em uma dinâmica colaborativa entre trabalhadores e cooperativas universitárias. A reflexão que propomos enfoca a produtividade cultural dessas iniciativas para contestar práticas e sentidos em torno dos processos de treinamento e treinamento para o trabalho implementado nessas experiências. O projeto materializou-se na elaboração da Plataforma de Co-mão-de-obra, uma interface on-line de acesso aberto que garante acesso aberto e irrestrito a conteúdos e propostas pedagógicas desenvolvidas com base na sistematização do aprendizado gerado por e para essas experiências de gerenciamento Coletivo de trabalho Assim, refletindo sobre essa iniciativa, procuramos deliberadamente abordar a questão formativa em espaços de trabalho recuperados e / ou conquistados por lutas populares, sempre enfatizadas pela dinâmica da desterritorializaçãoreterritorialização de suas práticas econômicas, sociais e culturais. Nessa tensão, colocamos a reflexão sobre o aprendizado derivado do trabalho da equipe com as cooperativas em 19 de dezembro e a Textiles I continuou refletindo sobre as contribuições dessa experiência colaborativa para repensar as modalidades de extensão universitária.

Palavras-chave: formação, plataforma digital, cooperativas, trabalho, desenho colaborativo.

Para citación de este artículo: Carenzo, S.; Fernández Álvarez, M.I.; Castronovo, A.; Gigliarelli, E. (2019). Extensión en Colabor: la producción de prácticas autogestionadas de formación para la gestión colectiva del trabajo. +E: Revista de Extensión Universitaria, 9(11), 151-170. doi: 10.14409/ extension.v9i11.Jul-Dic.8748. 


\section{Introducción}

En este artículo ${ }^{1}$ comparte resultados derivados de una experiencia de extensión universitaria crítica y reflexiva, que vinculó a trabajadoras/es de universidades públicas y cooperativas de trabajo que gestionan empresas recuperadas con el objeto de desarrollar metodologías y contenidos destinados a fortalecer prácticas autogestionadas de formación para la gestión colectiva del trabajo. ${ }^{2}$ Con esta última expresión queremos destacar que tanto los contenidos como las metodologías se derivan de la sistematización de la experiencia situada surgida de las cooperativas involucradas. Además, que estos contenidos y metodologías fueron diseñados para ser implementados en prácticas de formación organizadas "desde y para" estas y otras experiencias de gestión colectiva del trabajo, sin contar necesariamente con la participación de terceras partes "externas" a las propias experiencias. ${ }^{3}$

En primer término, esto implicó un proceso de reconocimiento y reconstrucción de aprendizajes significativos en cada iniciativa, incorporando incluso aquellos que, en un primer momento, nunca habían sido categorizados de ese modo por sus protagonistas. Una segunda instancia de trabajo colaborativo consistió en la elaboración de una propuesta pedagógica basada en materiales gráficos y audiovisuales que pudieran condensar su contribución a procesos formativos autogestionados capaces de desplegarse incluso por fuera de las experiencias en las que tuvieron lugar inicialmente. Luego, esta labor conjunta incluyó el diseño e implementación de una plataforma digital en línea (http://www.colabor.com.ar/) que hace las veces de repositorio interactivo, garantizando el acceso abierto e irrestricto a los materiales de formación elaborados. Esta última instancia cierra el ciclo iniciado con la identificación y sistematización de aprendizajes situados, con el desarrollo de una propuesta pedagógica destinada a nutrir experiencias de formación autogestionadas y que, a su vez, promuevan la sistematización de nuevos aprendizajes que alimenten el repositorio de la Plataforma Co-labor (PC) en forma continua. ${ }^{4}$

1) Quisiéramos agradecer a los/as evaluadores/as anónimos por los sustantivos comentarios a la versión original de nuestro texto.

2) Utilizamos esta denominación, gestión colectiva del trabajo, con el ánimo de evitar clasificar estas experiencias a partir de nociones predefinidas como autogestión o cooperativas que portan una carga valorativa previa. Lejos de proponer un concepto teórico alternativo, esta categoría descriptiva tiene la intención de señalar el carácter fragmentario, contradictorio, heterogéneo, múltiple y sobre todo en permanente construcción que encontramos al analizar estas experiencias (Fernández Álvarez, 2015).

3) Esta experiencia se enmarco inicialmente en el Proyecto $N^{\circ} 32$ "Caja de herramientas audiovisuales para la autoformación en cooperativas y otras experiencias de gestión colectiva del trabajo", financiado por el Programa Educación en Cooperativismo y Economía Social en la Universidad (Res. 77/2015 SPU-ME). El equipo coordinador del mismo integró representantes tanto de universidades como de cooperativas de trabajo quienes se encargaron de implementar el proyecto en su totalidad: Sebastián Carenzo (UNQ), Francisco Martínez y Leonor Litre (Cooperativa Textiles Pigüe), María Inés Fernández Álvarez (UBA), Gisela Bustos y Enrique Iriarte (Cooperativa 19 de Diciembre), Elisa Gigliarelli (Facultad Abierta-Sapienza Università di Roma), Fabián Pierucci (Alavio-FACTA), María de los Ángeles di Capua y Melina Perbellini (UNR), Alioscia Castronovo (UNSAM-SapienzaUniversità di Roma). Agradecemos las/os editores de la revista $E_{+}$, así como a las/os evaluadores anónimos por sus generosos comentarios que nos permitieron profundizar la reflexión propuesta en este artículo.

4) El desarrollo integral de la PC demandó casi dos años de trabajo e involucró instancias de investigación de campo (observación y entrevistas), elaboración de registros audiovisuales, así como una ardua tarea de post producción en función de la definición de los aprendizajes significativos y la elaboración de una propuesta pedagógica asociada a cada aprendizaje. A través de la PC, estos recursos pueden ser trabajados en línea, descargados o impresos, empleando dispositivos tales como computadoras de escritorio, portátiles, tabletas e incluso teléfonos celulares incluye una "guía de implementación" que orienta a sus usuarias/os para utilizar recursos audiovisuales que se incorporan como "disparadores temáticos" y de "materiales de apoyo conceptual y metodológico" que completan la propuesta pedagógica. 
Con el propósito de contribuir a la convocatoria de la revista $+E$, en cuanto a pensar la vinculación entre cultura y territorios desde la perspectiva de la extensión, enmarcamos las reflexiones derivadas de la iniciativa caracterizada previamente en términos de una práctica cultural vinculada a los procesos de formación y capacitación para el trabajo en el marco de experiencias de gestión colectiva del trabajo.

De manera de compartir una mirada retrospectiva y reflexiva sobre este proceso, organizamos el artículo en tres apartados. En el primero incorporamos una reflexión metodológica necesaria, en tanto el desarrollo de esta experiencia de extensión se realizó en forma integrada con nuestra trayectoria previa en investigación desde una perspectiva de etnografía colaborativa. Consideramos que este enfoque puede aportar sugerentes pistas metodológicas para una práctica de extensión que sea al mismo tiempo rigurosa y comprometida, razón por la cual creemos prudente darle un espacio específico. El segundo apartado presenta la elaboración conceptual efectuada a través de esta experiencia con la PC, sintetizada en una concepción particular de lo que entendemos por "aprendizajes", puesto que resultó un eje clave de nuestra práctica colaborativa y estimamos pertinente delimitarla y explicitarla. De modo complementario, recuperamos sintéticamente uno de los principales aportes teóricos que nos permitió conceptualizar estos aprendizajes por fuera de la matriz individualista y racionalista que focaliza solamente en las capacidades cognitivas formales. Siguiendo a Etienne Wegner (2001), destacamos el carácter colectivo de los procesos pedagógicos, lo identificamos, sistematizamos y reelaboramos en la PC, mediante el reconocimiento y énfasis de su ligazón con el involucramiento relacional con las contradicciones y ambivalencias del mundo vívido. En el tercer y último apartado analizamos dos contenidos incorporados a la PC y destacamos su carácter de aprendizajes vivos, es decir, el proceso de elaboración de estos materiales derivados de experiencias situadas para energizar una propuesta pedagógica capaz de alimentar prácticas autogestionadas de formación para el trabajo en otras experiencias. Finalmente, presentamos algunas reflexiones que tienen por objeto recuperar el potencial de la propuesta de etnografía colaborativa para promover una práctica de extensión vinculada a las luchas y demandas de los movimientos populares.

\section{Repensar la relación entre cultura(s), trabajo y territorio desde la práctica de la extensión universitaria}

Antes de continuar con el desarrollo de nuestro argumento, consideramos pertinente introducir algunas precisiones para explicitar nuestro enfoque sobre la relación entre cultura, trabajo y territorio, desde el cual analizamos la experiencia de construcción colaborativa de la PC.

En primer lugar, cabe señalar que, siguiendo la propuesta de Luis Reygadas (2002), abordamos la relación entre cultura y trabajo desplazándonos de una mirada dicotómica que contrapone una dimensión "simbólica" asociada a la "cultura" con otra dimensión "material" vinculada al "trabajo" en tanto práctica material y productiva. En contraste, Reygadas propone un enfoque relacional acerca del proceso de trabajo, entendiendo que, además de resultar modelado por condicionantes técnicos, sociales, económicos y políticos, constituye un espacio privilegiado de producción y transformación cultural, esto es, al producir, transformar y/o impugnar prácticas y sentidos asociados a la reproducción de la vida. Ahora bien, en tanto esta labor resulta territorialmente situada, recuperamos la definición de territorio y 
territorialidad elaborada desde los estudios contemporáneos de geografía humana. Si bien no trabaja desde la noción de territorio, el abordaje sobre la categoría de espacio propuesta por Doreen Massey (2012) nos permite señalar una primera cuestión de importancia a atender: el espacio geográfico no se limita al emplazamiento físico sino que remite al espacio relacional efectivamente apropiado por un grupo social para asegurar su reproducción. $Y$ de acuerdo con Haesbaert (2013), estas relaciones involucran el despliegue de relaciones de poder, siendo que el territorio es una dimensión del espacio, justamente aquella que focaliza en el modo en que se despliegan estas relaciones y establece asimetrías a nivel de los agentes sociales para crear, recrear y apropiar el espacio en tanto territorio. La noción de territorialidad enfatiza la importancia de atender a la actividad espacial de los agentes -expresada en términos materiales y simbólicos- capaces de garantizar la producción, apropiación y permanencia de estos territorios a partir de concepciones y prácticas espaciales en disputa (Gamba, 2012). De esta manera, los enfoques contemporáneos sobre territorio se caracterizan por atender a su dimensión multiescalar y multidimensional, dando lugar a configuraciones "flexibles y móviles" vinculadas a la producción de "territorios en red" o "multiterritorios", en cuya configuración resulta clave atender al rol de las Tecnologías de la Información y la Comunicación (TIC) en su doble inscripción, tanto para promover un creciente control y disciplinamiento como para desplegar procesos de resistencia y emancipación (Altshuler, 2013). Es entonces desde estas nociones de trabajo, cultura y territorio, que enmarcamos el desarrollo de la PC como un proceso para fortalecer prácticas de formación colectiva para el trabajo, elaboradas "desde y para" experiencias de gestión colectiva que desarrollan proyectos socioproductivos desde los márgenes del sistema capitalista. Se trata de experiencias variadas, desde empresas recuperadas a asociaciones de trabajadores "precarios" o "informales" que crean a partir de la práctica modalidades de "hacer juntas/os" en su doble inscripción política y productiva como dimensiones concomitantes, al tiempo que deben lidiar con lógicas políticas, financieras y culturales hegemónicas que amenazan cotidianamente su existencia y reproducción (Fernández Álvarez, 2016).

En segundo lugar, a través de la PC buscamos deliberadamente abordar la cuestión formativa en espacios laborales recuperados y/o conquistados a través de luchas populares, cuya mera existencia supone una interpelación al statu quo, y por lo tanto se encuentran siempre tensionados por dinámicas de desterritorialización-reterritorialización de sus prácticas económicas, sociales y culturales. Como señala Roberto Elizalde (2010), en este tipo de experiencias, la propia práctica cotidiana resulta el medio de comunicación y educación privilegiado para transmitir y educar desde otras lógicas y proyectos de vida que desplazan del modelo dominante. Para profundizar en esta idea, Anahí Guelman (2012) sostiene que en estas experiencias la formación para el trabajo excede los contenidos técnicos de carácter productivo, de gestión y de comercialización, para involucrar un conjunto de prácticas cotidianas existentes en los colectivos de trabajadoras/es, que comprende desde competencias requeridas para llevar a buen puerto delicadas dinámicas de negociación y articulación con otros colectivos y agencias del Estado, hasta la definición de criterios de incorporación de nuevas/os asociadas/os. El aspecto cultural y pedagógico vinculado al trabajo asume una perspectiva amplia y reconoce

"múltiples prácticas y formas de trabajo útil corridas de la lógica de las relaciones salariales típicas, de las relaciones de empleo, de la lógica de mercado y de la ganancia individual. Buscan un crecimiento de los trabajadores que se traduce en saberes: saberes 
técnicos, productivos, de gestión, organizativos, culturales, comunitarios y políticos. De allí, las preocupaciones educativas hacia los propios trabajadores pero no solo en la fábrica sino promovido y extendido a su contexto, hacia el territorio". (Guelman, 2012:76)

En tal sentido, los conocimientos, habilidades y disposiciones relacionados con estos aspectos usualmente considerados como "extraproductivos" también pueden entenderse en clave de instancias pedagógicas en sentido amplio que pueden trabajarse en términos de la formación para el trabajo en estas iniciativas.

Y el último aspecto a abordar en nuestra argumentación focaliza en la práctica de la extensión universitaria en términos de la sistematización y elaboración de contenidos y metodologías pedagógicos desarrollados por fuera de los marcos institucionales formales y no formales que trabajan en este campo (desde centros de formación sindical, escuelas técnicas, fundaciones, asociaciones civiles y, por supuesto, universidades). Como bien señala Natalia Baraldo (2010), al trabajar desde la extensión con experiencias organizadas desde y para movimientos populares, el desafío pasa por "rebasar" aquello que tradicionalmente se ha entendido como educación, proponiéndonos pensar otros principios o matrices educativas, recuperando el carácter educativo de las propias prácticas situadas al ser incorporadas en un ejercicio reflexivo más amplio. En el mismo sentido, coincidimos con Norma Michi, Diana Vila y Javier Di Matteo (2014) cuando plantean que no es lo mismo pensar la extensión en términos de fortalecimiento de procesos organizativos populares en conjunto con sus integrantes que hacerlo en términos de transferencia de conocimientos o servicios, que si bien pueden presentarse desde un genuino interés por la búsqueda de soluciones a problemas sociales "concretos" -incluso desde un sentido de "compromiso con la sociedad"-, pueden también hacer abstracción de la conflictividad y dar lugar a posiciones neutrales y descomprometidas con las luchas de sus protagonistas. Así, la experiencia desarrollada en torno a la PC, que recuperamos reflexivamente en este artículo, significó operar un desplazamiento de los formatos curriculares estándar, de los roles atribuidos docente-estudiante y del registro escrito como principal soporte pedagógico, que en general habían caracterizado las experiencias de articulación con equipos universitarios en el pasado, para explorar nuevas formas (más simétricas y colaborativas) de elaboración de conocimientos e implementación de experiencias de formación autogestionada. La experiencia de diseño y elaboración de la PC constituyó entonces una oportunidad para repensar nuestra propia labor de extensión universitaria, en el sentido de alentarnos a ampliar nuestros repertorios disciplinares para desarrollar prácticas de formación más creativas, colaborativas y territorialmente situadas. Así, nuestro artículo busca contribuir a la reflexión sobre las formas en que pensar la relación entre teoría y práctica en la extensión universitaria, señalando la relevancia de la noción de territorio en tanto categoría compleja y múltiple, lo que implica atender a las relaciones de poder que producen material y simbólicamente los espacios (Arzeno, 2018).

\section{Sobre el enfoque colaborativo que dio lugar a la elaboración de la plataforma}

Fue en ocasión del $V$ Encuentro Internacional de Economía de los Trabajadores desarrollado en Venezuela en el año 2015 cuando comenzamos a discutir la posibilidad de abordar la temática de prácticas autogestionadas de formación para la gestión colectiva del trabajo, 
un impulso que dos años después se materializó en la PC. La plataforma es básicamente una interfase en línea de acceso abierto y construcción colaborativa donde se producen y comparten contenidos y propuestas pedagógicas para el desarrollo de prácticas autogestionadas de formación en y desde experiencias de gestión colectiva del trabajo, tales como cooperativas de trabajo y otras iniciativas autogestivas. Si bien su acceso libre e irrestricto se habilitó en agosto de 2017, la construcción de la Plataforma sigue en proceso ya que se alimenta del aporte voluntario de nuevas experiencias, previo intercambio con el equipo coordinador conformado por trabajadoras/es de cooperativas e investigadoras/es de universidades nacionales. El carácter mixto de su coordinación se vincula con los orígenes de la iniciativa, compuesta por personas que veníamos participando activamente de los encuentros "La Economía de los/as Trabajadoras/es". En particular, el Segundo Seminario de la Red de Investigadores/as Latinoamericanos/as en Empresas Recuperadas y Cooperativas de Trabajadores/as, realizado en la ciudad de Córdoba en 2016, donde avanzamos en una lectura compartida respecto de la cuestión "formativa" en el marco de estas experiencias que podría ser sintetizada del siguiente modo:

- En particular, el crecimiento de cooperativas de trabajo en poco más de una década resulta muy significativo, lo cual ha sido recuperado y analizado por una heterogénea y abundante literatura.

- Sin embargo, la ponderación de estas iniciativas en función de sus aportes cualitativos - tanto en términos conceptuales como metodológicos- a los procesos de gestión colectiva del trabajo es menos frecuente.

- En el cotidiano de las experiencias abundan los formatos de "capacitación" sobre "cooperativismo" que articulan contenidos genéricos y universales, desde un modelo pedagógico marcadamente curricular basado en el supuesto de que la adquisición de conocimientos se realiza en función del avance acumulativo y lineal.

- Estos materiales son habitualmente elaborados por "técnicos", "expertos" o "profesionales" con limitada participación de trabajadoras/es en el diseño de contenidos y herramientas pedagógicas (formatos y soportes pedagógicos, metodologías propuestas para su discusión y elaboración).

- Los escasos materiales sistematizados desde espacios de investigación y docencia universitaria con participación de trabajadoras/es privilegian un registro textual escrito más que oral, lo cual suele limitar el uso práctico de estos aprendizaje por fuera de ámbitos específicamente destinados a la docencia-investigación que los originó.

- Resultan aún más escasas las instancias donde estos saberes y conocimientos hayan sido sistematizados por sus protagonistas para luego ser codificados en dispositivos de autoformación y construcción de herramientas pedagógicas que promuevan su (re)aplicación y circulación horizontal.

- En general, las prácticas formativas existentes no exploran posibilidades de promoción de la autoformación, que puede dar lugar a procesos de construcción de conocimiento desde una lógica modular y flexible antes que lineal y de tipo curricular con contenidos preestablecidos.

- Menos exploradas incluso han sido las herramientas interactivas digitales basadas en el uso pedagógico de plataformas en línea. 
En función de esta lectura compartida comenzamos a diseñar en forma conjunta los lineamientos básicos de la PC: proveer materiales para la formación autogestionada elaborados sobre la base de la sistematización de los aprendizajes generados en las experiencias de gestión colectiva del trabajo, con un activo involucramiento de trabajadoras/es en su diseño y elaboración, priorizando el empleo de recursos audiovisuales por sobre el texto escrito y desde un soporte digital e interactivo en línea. Nos interesa en este sentido destacar la idea de codiseño como principio que organizó el trabajo llevado adelante en torno a la elaboración de la PC desde un primer momento, el cual incluyó qué aspectos serían trabajadores prioritariamente en los contenidos sobre los que se desarrollarían los materiales.

Este principio organizador de la propuesta responde a que la iniciativa se enmarcó en un trabajo de etnografía colaborativa (Hale, 2006; Solano y Speed, 2008; Rappaport, 2007), una práctica que tiene larga data en la antropología, específicamente en América Latina (Jimeno, 2008). Esta perspectiva parte de una modalidad de producción de conocimiento en la cual el espacio del trabajo de campo se considera como una instancia de producción teórica conjunta (Rappaport, 2007), es decir, un ámbito de creación conjunto de categorías de análisis que articula los ejes de investigación con problemas derivados de la práctica cotidiana y recoge el trabajo llevado a cabo con las organizaciones a partir de la generación de instancias de discusión, intercambio y reflexión con estos colectivos (Fernández Álvarez y Carenzo, 2012). Esto implica tomar distancia de lo que algunas autoras Ilaman "modelo extractivista" (Speed y Solano, 2008), según el cual el trabajo de campo es una instancia de recolección de datos y sobre esa base el/la investigador/a produce conocimiento que luego transfiere a la sociedad, de manera tal que investigación y transferencia suponen dos instancias escindidas. El enfoque colaborativo habilita un camino donde el conocimiento es coproducido con aquellas y aquellos con quienes llevamos adelante la investigación e identificamos en conjunto problemas y soluciones, fundamento sobre el que se diseñan instancias de intervención, de manera tal que investigación y transferencia son instancias entrelazadas (Carenzo y Fernández Álvarez, 2017). Se trata, en consecuencia, de un enfoque afín a las perspectivas críticas y reflexivas sobre la extensión universitaria que responde a una lógica de integralidad en cuanto a diálogo de saberes que suelen definirse en términos escindidos como "académicos" y "populares" (Tomassino y Cano, 2016; Tommassino y Stevenazzi, 2016). Al igual que la investigación colaborativa, la extensión crítica recupera aportes de la educación popular y la Investigación Acción Participativa, enfoques desarrollados en América Latina a partir la obra de Paulo Freire y Orlando Fals Borda, respectivamente.

Así, la PC es un resultado derivado de la red construida en los encuentros y las dinámicas de formación autogestionada elaboradas desde el territorio, invirtiendo la lógica que suele organizar las relaciones entre universitarios y experiencias de gestión colectiva del trabajo. En lugar de trabajar desde las agendas particulares que cada equipo universitario desarrollada con tal o cual cooperativa y diseñar un dispositivo localizado en el espacio académico, decidimos poner en común los recursos, relaciones y habilidades, para construir la PC como un proyecto mixto, colectivo y deslocalizado. De este modo desafiamos las lógicas de acumulación y competencia sumamente frecuentes en el mundo académico, pero además apostamos a producir contenidos "situados", en tanto se derivan de la experiencia vívida de y en cooperativas en particular, pero también "transversales", ya que su valor está asociado a las posibilidades de ser reaplicados y recontextualizados en otras experiencias, incluso 
de carácter muy disímil. En este sentido, un principio fundamental en la construcción de la Plataforma consistió en un ejercicio de deslocalización y reterritorialización de las prácticas de formación al situar el diseño, la reflexión y ejecución de las mismas en el seno de las experiencias de gestión colectiva.

Así, la PC como proyecto en común pudo ser realizado aprovechando las potencialidades y lidiando con las tensiones, mediante la obtención de financiamiento público para desarrollar una novedosa práctica de articulación y colaboración entre trabajadoras/es de universidades y experiencias autogestivas. En lo que sigue puntualizaremos brevemente los aspectos conceptuales de aquello que entendemos por "aprendizajes" en esta experiencia, para luego ejemplificar con el trabajo efectuado con las cooperativas 19 de Diciembre y Textiles Pigué en la Plataforma.

\section{Conceptualización de los "aprendizajes"}

Si retomamos la potencialidad/desafío que supone ponderar la particularidad situada de un determinado aprendizaje, al tiempo que lograr interpelar otras experiencias, incluso muy disímiles, nos planteamos dos preguntas que atraviesan toda práctica de gestión colectiva y que nos permiten especificar por qué tomar la cuestión de los aprendizajes como perspectiva:

-¿Cómo aprendemos?: esta pregunta pone el foco en la especificidad de las prácticas de aprendizaje derivadas de la propia experiencia. Se trata un aprendizaje fuertemente dependiente del contexto en el cual se realiza, enraizado en la práctica cotidiana, cuya circulación y pregnancia depende del hecho de compartir/participar de la actividad y luego de su comunicación oral, no como un "aprendizaje" en sí mismo, sino como parte de una experiencia vivida en diversos formatos narrativos (historias, eventos, chistes, anécdotas). - ¿Cómo resignificamos otros aprendizajes?: este interrogante focaliza en la apropiación y adecuación de conocimientos y formas de hacer derivados de "otras" experiencias e iniciativas, incluyendo instancias de "capacitación" formal. Este punto enfatiza el carácter heterogéneo de los aprendizajes elaborados, que de ninguna manera resulta restringido a los límites de cada experiencia sino que se nutre de diversas fuentes, códigos, espacios y soportes.

Ambas preguntas lanzadas inicialmente por Fabián Pierucci (Grupo Alavío y FACTA) en uno de los encuentros para discutir objetivos, alcances, orientación y factura de la Plataforma, conectan con algunas referencias conceptuales claves que veníamos trabajando desde los equipos universitarios y que sintetizamos a continuación.

Por una parte, siguiendo a James C. Scott (1998), abordamos el "conocimiento práctico" (métis) como aquel que resulta contextual, situado y adecuado. Contextual en la medida en que organiza una lectura de detalles, elementos, señales que resultan significativas con relación a cierta acción. Situado en tanto esa acción sucede en un espacio-tiempo determinado, de allí que Scott también se refiere a este conocimiento como "vernacular", es decir, vinculado específicamente a un lugar. Adecuado en tanto provee un tipo de solución "a mano" que, sin ser compleja o costosa, admite adaptaciones prácticas a situaciones y contextos cambiantes. Finalmente, Scott remarca la íntima relación entre el desarrollo de estas habilidades y disposiciones que caracterizamos como aprendizajes y la práctica de la acción de 
la cual se derivan (por eso son "difíciles" de enseñar en abstracto). Así, para responder a la pregunta ¿cómo aprendemos?, podemos señalar que, en términos de métis, no basta con intelectualizar las situaciones y analizarlas en función de diseccionar elementos y factores, tal como procede el método científico moderno. Por el contrario, será clave sentir, pensar y hacer desde la práctica para entonces aprender a sentir, pensar y a hacer tal o cual cosa y, de esta manera, incorporarlo. No se aprenden rutinas de procedimientos opacos y estandarizados, sino formas de hacer sobre la base de algunas orientaciones, pero por sobre todo mixturando tanto la experiencia y el dominio de la práctica ya incorporada como también la flexibilidad requerida para ajustar e improvisar sobre la marcha (ya que en general operamos sobre circunstancias cambiantes). Por eso otra de sus características es la dificultad para codificarlo en términos escritos o incluso orales. ${ }^{5}$

Otra referencia clave es aportada por Etienne Wenger (2001), quien aborda estos procesos desde una teoría social del aprendizaje sustentada en su conceptualización de las "comunidades de práctica", por la cual propone desplazamientos respecto de una noción más generalizada de "aprendizaje". En primer término, evitamos considerar a los aprendizajes como el desarrollo de potencialidades específicamente humanas encarnadas en un sujeto abstracto universal. En contraste, destaca el carácter iteractivo, relacional e intersubjetivo de los procesos de aprendizaje. No aprendemos como individuos aislados a través de un proceso que se da exclusivamente "en nuestra mente", sino que aprendemos-con-otros a partir de nuestro involucramiento relacional con el mundo vívido y su trama de vínculos. Un segundo desplazamiento opera con referencia a aquellas nociones que lo definen como un proceso intrínsecamente ligado la práctica de enseñanza, organizado a partir de progresión lineal de una currícula, y por ende jalonado con un "principio" y un "fin". Por el contrario, sostenemos que los procesos de aprendizaje son continuos, no lineales y muchas veces recursivos. Así, también podemos responder al ¿cómo aprendemos? señalando que lo hacemos al formar parte de tales comunidades de prácticas (por ejemplo, las que se dan en una cooperativa). En palabras de Wenger: "Esta participación no solo da forma a lo que hacemos, sino que también conforma quienes somos y cómo interpretamos lo que hacemos" (2001:22). Incluso, en cada cooperativa se organizan múltiples comunidades de práctica en función de la tarea específica, de la sociabilidad, de los encuadres políticos, de los compromisos religiosos, etc. Son tan "informales como omnipresentes" y de algunas somos miembros muy activos y de otras más periféricos. Asimismo, podemos señalar que desde esta perspectiva los aprendizajes se expresan simultáneamente en tres planos:

- para los individuos, significa que el aprendizaje consiste en participar y contribuir a las prácticas de sus comunidades;

5) El filósofo Michel Polanyi (1983) había anticipado un planteo similar hacia mitad de los 60, dando pie a la distinción entre conocimiento "tácito" y "codificado", que fuera muy trabajada posteriormente por autores como Nonaka y Takeuchi (1995) para abordar la "gestión del conocimiento" en organizaciones como una dimensión clave del proceso de innovación. Si bien existen similitudes entre estos planteos y el desarrollado por Scott, preferimos conservar esta última propuesta por dos razones. Por una parte, en tanto no hace hincapié en la cuestión de la codificación o no (y, por ende, comunicabilidad) del conocimiento como factor principal para su definición. Luego porque, a diferencia de los otros planteos, que se limitan a señalar el anclaje "cultural" del conocimiento tácito pero sin profundizar a qué refiere esto, Scott profundiza en el tipo de relación con prácticas y sentidos elaborados colectivamente como forma de procesar y hacer disponible la experiencia. 
- para las comunidades, significa que el aprendizaje consiste en refinar su práctica y garantizar nuevas generaciones de miembros;

- para las organizaciones, significa que el aprendizaje consiste en sostener interconectadas las comunidades de práctica, a través de las cuales una organización sabe lo que sabe, y en consecuencia llega a ser eficaz y valiosa como organización (Wegner:25).

En conjunto, estas referencias conceptuales nos permitieron operacionalizar una noción de aprendizaje en función de los objetivos que nos propusimos para la PC y que puede sintetizarse en dos orientaciones claves. En primer término, partimos de valorizar y jerarquizar los aprendizajes elaborados en las propias experiencias de gestión colectiva del trabajo en términos de un "conocimiento práctico", que va mucho más allá del expertise ligado a la mera repetición rutinaria de una tarea e involucra formas compartidas de sentir, pensar y hacer. En segundo término, al recuperar la noción de "comunidades de práctica", ponderamos la relación entre los aspectos cognitivos, identitarios y hermenéuticos derivados de la acción situada (involucrarse en la actividad). Sin embargo, cabe aclarar que esto no significa atribuir una identidad social y/o cultural predefinida a los aprendizajes de estas experiencias solo por el hecho de haber sido elaborados en tal o cual cooperativa pensada como una "comunidad" encerrada en sí misma. En cambio, la noción de "comunidades de práctica" hace foco en el hacer $y$, en cuanto tales, estas comunidades pueden estar habitadas por sujetos heterogéneos, portadores de diversos conocimientos y experticias. Recuperando (y subvirtiendo un poco) la célebre frase de Clifford Geertz, no nos interesa recuperar aprendizajes de cooperativas sino aquellos aprendizajes elaborados en cooperativas. Así, las "comunidades de práctica" con las que trabajamos están conformadas mayoritariamente por trabajadoras/ es de cooperativas, pero los aprendizajes elaborados involucran también a otros sujetos que puede ser profesionales universitarios, militantes y activistas, así como habitantes de los barrios donde se localizan o colegas de otros espacios con quienes articulan, en términos políticos, comerciales, solidarios.

A este respecto, cuando hablamos de sistematizar aprendizajes, nos referimos a documentar un proceso abierto, lleno de matices y contradicciones, más que a relevar un repertorio cristalizado de soluciones efectivas a problemas puntuales. Destacamos entonces la creatividad puesta en juego en la práctica cotidiana, incluyendo desde luego estrategias y metodologías que se dieron para resolver cuestiones prácticas, abrir espacios de discusión y debate, desarrollar nuevas prácticas y procesos; pero también, como propone Fernández Álvarez (2016), dando cuenta de la forma contingente y situada en la cual se definen "aciertos" y "errores", con marchas, contramarchas y aun "fracasos", a los cuales esta perspectiva les otorga un alto valor en términos pedagógicos. Esto abre la posibilidad de poner en común una serie de preguntas que surgen de la colaboración en torno a los cambios, las transformaciones y los desafíos que los procesos de lucha colectiva enfrentan y contribuyen a construir, así como a las formas en que se producen nuevos saberes, valores y disputas.

Ahora bien, una derivación práctica de esta formulación es que, en términos generales, esta rica experiencia acumulada es fácilmente reconocida al interior de los colectivos en cuanto a "experticia" pero no en términos de "aprendizaje" en el sentido de reconocer su potencia pedagógica frente a otros colectivos que puedan recuperarlo, resignificarlo y reaplicarlo. De allí que el mismo desarrollo colaborativo de la PC activó este (auto)reconocimiento 
y promovió la sistematización de estos conocimientos para potenciar la procesos de transferencia horizontal de los aprendizajes. Para esto resultó clave trabajar con metodologías de soporte y codificación que trataran de respetar lo más posible el carácter contextual, situado $y$ adecuado, en el cual se elaboran y transfieren estos modos de sentir, pensar y hacer. Esto es, si en una cooperativa uno aprende mirando y participando, antes que leyendo y estudiando, los materiales que nos propusimos fabricar debían ser lo más análogos posibles a la experiencia vívida. Por eso decidimos minimizar los textos escritos y priorizar la confección de piezas audiovisuales cortas y modulares, que facilitaran un uso más cotidiano y creativo de estos contenidos y que, al estar organizadas en una plataforma digital, contribuyeran a interesar a las generaciones más jóvenes mucho más habituadas a manejar contenidos en Internet y redes sociales.

\section{Aprendizajes vivos en Plataforma Colabor}

A continuación, vamos a hacer referencia a dos aprendizajes que elaboramos en conjunto con las/os compañeras/os de las cooperativas 19 de Diciembre y Textiles Pigüé, especificando la metodología de sistematización de los aprendizajes y el diseño de una propuesta pedagógica específica destinada a dinamizar prácticas de autoformación en otras experiencias.

\section{Cooperativa 19 de Diciembre: "abrir" la cooperativa como desafío y potencialidad}

Esta fábrica autopartista situada en Villa Ballester fue recuperada por sus trabajadores en diciembre de 2002, en plena crisis económica, tras su abandono y quiebra por parte de sus patrones. En ese contexto, con el apoyo de vecinos del barrio, de las asambleas vecinales, de organizaciones populares y militantes políticos, los trabajadores decidieron llevar adelante una dura y difícil lucha para recuperar su fuente de trabajo y asumieron el desafío de reorganizar la producción bajo autogestión. Hoy, tras 15 años de lucha y trabajo, es reconocida no solo por la rigurosidad de su labor productiva, sino también por impulsar iniciativas solidarias con otras "recuperadas" y organizaciones sociales, fortaleciendo una densa trama sociocomunitaria a nivel local. Para sus integrantes, la construcción de una fábrica "abierta" al barrio, tiene que ver con "devolver" el apoyo que recibieron en los momentos más duros de la lucha. Así, la fábrica autogestionada por la cooperativa coexiste con una Biblioteca y un Bachillerato Popular especializado en cooperativismo y su inserción a nivel territorial en diferentes espacios de articulación local: Mesa de Empresas Recuperadas por sus Trabajadores de San Martín, Mesa Reconquista, que reúne movimientos sociales y cooperativas del partido, articulación con la Universidad Nacional de San Martín y con el Municipio homónimo. Es por esto que sus integrantes se refieren a sí mismos como "Espacio Popular 19 de Diciembre" (EP19D), denominación que caracteriza la densa red de relaciones en el cual está entramada la cooperativa. Este espacio desborda los contornos de la cooperativa como proyecto y de la fábrica y evidencia la multiplicidad de ámbitos en que se despliega su labor, entendida como práctica creativa, colaborativa y transformadora que desde las luchas y sus conexiones abre y reconfigura lo urbano "desde abajo". Al mismo tiempo, permite reparar en aquellos saberes, conocimientos, prácticas que se desarrollan en estos procesos como recursos para reorganizar, crear y reinventar una organización colectiva del espacio urbano constituido por cuerpos, afectos, deseos, rituales, creencias, valores compartidos. 


\section{Problematizando el principio "Compromiso con la comunidad"}

Una de las primeras cuestiones que discutimos en el diseño de la PC giraba en torno a la organización temática y conceptual de aquellos aprendizajes que comenzábamos a identificar y sistematizar. Buscamos desplazarnos de criterios "formales" (sector, rubro, conformación, etc.), ya que sosteníamos que los aprendizajes - tal como los veníamos definiendo- eran transversales a experiencias muy heterogéneas. Fue así que José Pereyra (Cooperativa Los Chanchitos) propuso trabajar con los "siete principios del cooperativismo", ya que se trataba de un tópico frecuente en las capacitaciones "estándar" a las que accedían las cooperativas. Esta propuesta dio lugar a un interesante debate en torno al carácter normativo con el cual se aborda la cuestión de los "principios". Como resultado, acordamos como propuesta problematizar los siete principios desde aquellos aprendizajes elaborados en la práctica concreta de las experiencias que se relacionan con ellos identificando sus dilemas, desafíos y contradicciones.

Así, los aprendizajes derivados del EP19D se nos presentaban como una oportunidad clave para repensar la cuestión de las "fronteras" en las experiencias de gestión colectiva del trabajo. En cuanto al $7^{\circ}$ principio, que se focaliza en la relación entre cooperativa/comunidad, nos preguntábamos: ¿dónde empieza y termina cada una de estas entidades? Y más aún: ¿empiezan y terminan en algún hito o espacio? Aquello que evidenciaba la experiencia del EP19D era que estas fronteras no solo involucraban múltiples bordes y contornos sino que además eran continuamente redefinidos, de manera tal que el "adentro" y el "afuera" de la cooperativa se iba reconfigurando, transformaba subjetividades y generaba nuevos procesos sociales, laborales, políticos y culturales. A modo de ejemplo, la "salita del barrio" (Centro de Atención Primaria Villa Esperanza) está localizada a unas 15 cuadras de la fábrica; sin embargo, la relación con este espacio es cotidiana y permanente, no solo porque muchos trabajadoras/es de la fábrica se atienden allí, sino porque los profesionales que la integran participan en actividades de formación y militancia con estas/os trabajadoras/es pero también con las/ os docentes del bachillerato. En términos de problematizar el $7^{\circ}$ principio, podemos decir que la construcción de un vínculo con "la comunidad" difiere según las formas específicas de articulación en cada caso, pero siempre implica la redefinición de espacios, tiempos, estrategias, tareas, responsabilidades individuales y colectivas al interior de la cooperativa. El desarrollo de los materiales audiovisuales ${ }^{6}$ y pedagógicos que elaboramos a partir de ella evidencia que, más allá de cómo estén definidas y practicadas las "fronteras" entre cooperativa/comunidad, el propio ejercicio de objetivarlas y reflexionar sobre ello resulta un aporte significativo para evitar asumir su existencia de manera naturalizada, invitándonos a repensarlas creativamente.

La metodología utilizada para recuperar y sistematizar este aprendizaje consistió la realización de un taller en el que elaboramos "mapeo colectivo" de las diferentes experiencias que conforman el núcleo del EP19D. El taller propició un momento de diálogo, reflexión y formación entre diferentes subjetividades (incluyendo los investigadores) que posibilitó poner en común debates en torno a las vinculaciones, los límites y los aprendizajes que surgieron desde la articulación territorial de la cooperativa. De esa forma, el proceso de elaboración colectiva de un mapa propició reflexiones acerca de lo que estaba en juego en el EP19D y

6) Se puede acceder al material desde el siguiente enlace: https://www.youtube.com/watch?v=glOOkkKfgSs\&feature=youtu.be 
sus articulaciones y movilizó sensaciones, curiosidades, pasiones, afectos, memorias, desafíos y tensiones que, tal como destacaba Gisela Bustos - socia de la cooperativa y docente del bachillerato-, son herramientas claves para enfrentar la urgencias de la actual coyuntura económica, social y política, sin perder de vista la necesidad que existe a nivel de los colectivos autogestionados por encontrar tiempos y espacios para discutir, debatir y proyectar colectivamente. A este respecto, repensar y problematizar desde la práctica las fronteras, internas y externas, del propio espacio, permite complejizar las imágenes y los imaginarios tanto de los protagonistas como del "afuera" es un ejercicio colectivo que abre espacios de renovación estratégica. La construcción de un mapa colectivo hace factible además desarrollar estrategias nuevas para enfrentar las problemáticas territoriales y transformar la propia experiencia cooperativa en un contexto caracterizado por el despliegue de procesos de individualización, subjetivación y descomposición de la base comunitaria operados por las políticas del Estado y de las finanzas (Gago, 2017). Permite repensar y poner en juego las estrategias de construcción de redes, vínculos, formas de resistencia a nivel socioespacial y transformación política comunitaria y popular ponderando —como sostiene Gutiérrez Aguilar (2015) - pautas de respeto, colaboración, dignidad y reciprocidad, no exentas de tensiones, que contribuyen a conceptualizar la dimensión multidimensional y multiescalar del territorio en el marco de procesos de producción y transformación socioespacial.

\section{Claves del aprendizaje y propuesta para el ejercicio de autoformación en la PC}

Como resultado del taller de mapeo comunitario en el EP19D, que se puede visualizar en la Plataforma, ${ }^{7}$ puntualizamos dos claves del aprendizaje para replantear desde la experiencia práctica los desafíos del principio cooperativo del "compromiso con la comunidad":

a) El "compromiso con la comunidad" supone modificar fronteras y transformar el espacio-tiempo de la cooperativa: el papel de vecinos y organizaciones en solidaridad con el proceso de recuperación de la fábrica fue fundamental y por esta razón se decidió destinar una parte de la fábrica para actividades abiertas a la comunidad. En 2006 se abrió el Bachillerato Popular y en 2012 la Biblioteca, cuya presencia ha modificado el uso de diferentes áreas de la fábrica y las formas de habitar el espacio. La apropiación por parte de estudiantes, vecinos y docentes, modifica profundamente el sentido de la recuperación que, además de garantizar puestos de trabajo digno, habilita servicios y lugares de sociabilidad alternativa, autogestionada y colectiva, impulsa procesos de educación popular y crea nuevos espacios culturales a través de la organización de festivales. Se trata de una profunda modificación que a lo largo de los años redefinió, puso en tensión y modificó las fronteras entre "el adentro" y "el afuera" de la fábrica, la administración y los trabajadores, el mercado y la cooperativa, lo que es considerado trabajo y lo que no, educación y militancia, formación y trabajo, lo individual y lo colectivo.

b) El "compromiso con la comunidad" supone sostener un proceso cotidiano que transforma las subjetividades y los territorios: la puesta en acto de este principio no es un atributo dado del trabajo cooperativo, como aparece en los manuales del cooperativismo, sino que tiene que ser elaborada específicamente dentro de cada colectivo en circunstancias 
y contextos territoriales diferentes, variables y múltiples. Se trata de una intencionalidad que se configura sobre la base de distintas variables y que se modifica con relación a procesos de lucha, de construcción y de subjetivación, que son parte del proceso mismo de construcción de ese "compromiso con la comunidad". Al mismo tiempo, supone enfrentar tensiones específicas, por ejemplo, aquellas ligadas a la participación de sus integrantes en tareas que exceden el estricto límite del desempeño económico y el espacio productivo de la fábrica, lo cual exige no solo reconocer estas tareas como parte del "trabajo", sino evidenciar su importancia para sostener y energizar el proyecto cooperativo más amplio en el cual se inserta cada experiencia en particular. Son procesos que tensionan las propias subjetividades en el marco de dinámicas colectivas que enfrentan las complejidades propias de llevar adelante la lucha en múltiples planos interrelacionados. Desde la fábrica y los proyectos allí alojados (Biblioteca/Bachillerato) que integran el EP19D, hasta su vinculación con el territorio en términos más amplios, involucrando ámbitos de relaciones sociales, políticas y laborales diferentes (Mesa Reconquista/Mesa de ERT de San Martín/Salita/UNSAM/Municipio), hasta relaciones nacionales e internacionales que en esos dos años se han ido construyendo. Emerge así en el mapeo, a la vez colectiva y singularmente, un conjunto de posibilidades de imaginación política en las cuales se componen dinámicas y procesos de subjetivación, sus dificultades, esfuerzos y los logros en el marco de un despliegue de agencias múltiples y que tensionan (o que buscan hacerlo) las relaciones de poder tanto en la organización del trabajo como en el territorio. Esto permite asimismo interrogar la eficacia política y productiva de los procesos de gestión colectiva del trabajo como actores clave de la producción de lo común desde su capacidad de conjugar la construcción de infraestructuras sociales con la sistematización de saberes desde y para la lucha y la transformación social en el marco del despliegue de una trama urbana comunitario-popular (Gutiérrez Aguilar, 2015).

En términos del ejercicio de autoformación, la propuesta giró en torno al taller de mapeo colectivo, en tanto práctica colectiva que posibilita subvertir el lugar de enunciación en cuanto a los relatos sobre la relación entre cooperativa/territorio. El ejercicio permite generar una discusión colectiva respecto de los espacios y proyectos que se construyen vinculándose con la comunidad, visibilizando los esfuerzos, las tareas, la labor silenciosa, minuciosa y cotidiana que hace factible su desarrollo. El compromiso, los vínculos, las redes políticas y sociales que se construyen son los resultados y constituyen a la vez las condiciones de posibilidad del crecimiento de la experiencia y de su reproducción, por medio de la construcción de relaciones con la comunidad en múltiples escalas y espacios. El ejercicio busca instalar la discusión en lo que hace a la importancia y productividad política de estas tareas, desdibujar e redefinir desde perspectivas y percepciones distintas las fronteras entre el adentro y el afuera de la cooperativa, repensar colectivamente la importancia de estos procesos y, finalmente, lograr un proceso de apropiación colectiva de los entramados que ese vínculo genera y admite, fortaleciendo el compromiso y la participación de sus asociados.

\section{Cooperativa Textiles Pigué: trabajar con nuestros prejuicios}

A mediados de 2001, la antigua empresa manejada por la familia Backellián entró en convocatoria de acreedores, con el peso de una deuda de varios cientos de millones de pesos. 
La quiebra de esta empresa Gatic SACIFIA dejó en la calle a miles de trabajadoras/es. Aquellas/os que formaban parte de la una de las fábricas localizada en la localidad de Pigué (sur de la provincia de Buenos Aires) se encontraron privados de su fuente de empleo y en situación de acreedores, por lo cual decidieron tomar la planta y conformar una Cooperativa de Trabajo para continuar la actividad de las instalaciones. Los primeros años fueron duros ante las dificultades para volver a desarrollar la producción, mantener su fuente laboral y ganar una posición respetada y valorada positivamente en el mercado. En la actualidad, Textiles Pigüé es uno de los más pujantes ejemplos de empresa recuperada por los trabajadores: los 60 que arrancaron la cooperativa se transformaron en 145, recrearon un mercado para sus productos, sumaron jóvenes profesionales, incorporaron y renovaron máquinas, participaron de las luchas sociales y políticas de la región y del país, bregaron incansablemente hasta que por fin consiguieron escriturar la fábrica y se convirtieron en administradores de miles de microcréditos para la región, entre muchas otras cosas que jalonaron el progresivo desarrollo de la cooperativa. Esta dinámica de crecimiento de la labor productiva de la fábrica,así como de expansión hacia otras actividades de índole sociocomunitarias, determinaron la necesidad de incrementar el número de trabajadoras/es asociadas/os. Tal como sucede en la mayoría de las cooperativas, el ingreso de nuevos socios/as se basa en la existencia de lazos preexistentes (parentesco, vecindad, etc.). En general, quien se incorpora es, de alguna manera, "recomendado" por alguien que ya es socio. Esta condición lo ubica en una "lista de espera" que, de acuerdo con los perfiles requeridos, va organizando la posible incorporación como asociado. Sin embargo, existe una excepción generada desde el propio Consejo de Administración. Una forma en la cual este mecanismo es "salteado" para promover una incorporación directa y sin vínculo previo que tensiona esta lógica del ingreso sustentado en relaciones de afinidad que responde al objetivo de llevar a fondo prácticas de solidaridad. Esta "excepción" consiste en habilitar la incorporación como asociados/as a compañeros/as jóvenes que vienen de transitar alguna experiencia de institucionalización derivada de problemas con la justicia, consumos problemáticos, etcétera.

\section{Problematizar el principio "Membresía abierta y voluntaria"}

Todo lo que se genera alrededor de este mecanismo de excepción es justamente lo que produce el aprendizaje que se deriva de esta experiencia y que nos permite "problematizar desde la práctica" este primer principio del cooperativismo. Básicamente, porque nos señala que los prejuicios sobre los que operan discriminaciones de distinto grado y complejidad nos atraviesan como sujetos y como colectivos, aun en aquellos que están organizados en base a prácticas de solidaridad y compromiso con la comunidad como las cooperativas. En esta oportunidad, la instancia de incorporación de nuevos socios se vuelve reveladora del modo en el cual estos prejuicios que existen en forma latente se materializan y hacen presentes en la práctica cotidiana. Específicamente del aprendizaje generado en la Compañía Textiles Pigüé (CTP), ${ }^{8}$ se deriva que la incorporación de nuevos integrantes atendiendo al principio de no discriminación no puede considerarse como "dada", hay que construir herramientas para volverla efectiva, para trabajarla con el resto de los compañeros/as. Así, la decisión de 
incorporar a personas sobre las cuales cae el peso de un estigma social, pese a representar un claro acto de solidaridad y coherencia, no puede llevarse a la práctica sin preparar al entorno que deberá recibirlas. En términos de la problematización del principio, la experiencia desarrollada en CTP resulta tremendamente reveladora, porque nos lleva a considerar situaciones paradojales que expresan/espejan prejuicios mucho más leves y (aparentemente) superfluos o banales. Como señalamos, en CTP decidieron incorporar a personas "estigmatizadas" como parte de una "acción concreta" en pos de ejercitar aquello que Francisco Martínez denomina "política del compartir". En este caso puntual, "compartir" refería a dar la oportunidad a una persona que había atravesado circunstancias difíciles con la justicia (con la consecuente estigmatización en un pueblo) para ocupar un puesto de trabajo construido desde la autogestión y rehacer su vida.

Como metodología para recuperar y sistematizar este aprendizaje, trabajamos con la "historia de vida" basada en el experiencia de Christian, un pibe a quien le tocó atravesar una infancia dura cargada de privaciones materiales pero sobre todo afectivas. Transitó la adolescencia desde los márgenes de un pueblo chico, donde los "errores" de juventud pueden garantizar un juicio duro y estigmatizado, difícil a sacar de encima. En este contexto, Christian se acercó al programa Envión (Ministerio Desarrollo Social, provincia de Buenos Aires) que desarrolla acciones de acompañamiento para niños y adolescentes que atraviesan alguna situación de vulnerabilidad y que venía articulando fuertemente con la CTP. Allí forjó una sólida relación con William Wanlesberg, tutor del Programa y socio trabajador en la Cooperativa con la que el Programa Envión llevó adelante una experiencia de pasantías orientadas a la formación y acceso al primer trabajo. Este proceso no fue lineal, estuvo jalonado por numerosas marchas y contramarchas pero finalmente dio resultado en tanto Christian logró ser incorporado como socio pleno. El punto fuerte del aprendizaje basado en la historia de Christian y su potencia para problematizar el principio tiene que ver justamente con resaltar el hecho de que no será solo Christian quién deba "prepararse" para la nueva experiencia, sino también las/os compañeras/os que aceptaron solidariamente "darle una oportunidad".

\section{Claves del aprendizaje y propuesta para el ejercicio de autoformación en la PC}

Este aprendizaje nos muestra la importancia de respetar el carácter progresivo de este proceso: tener en cuenta los tiempos y ritmos particulares a través de los cuales se acomoda a tareas y horarios, etc. En tal sentido, destaca la necesidad de pensar no solo en lo que Christian puede/hace o no puede/hace, sino en lo que el resto del colectivo puede/hace o no puede/hace. Así, destacamos dos claves de este aprendizaje:

a) La incorporación de nuevos integrantes atendiendo el principio de no discriminación no puede considerarse como "dada", hay que construir herramientas para volverla efectiva en la práctica cotidiana: la experiencia de CTP resulta sumamente pedagógica al respecto, porque al organizarse sobre un caso "extremo" permite evidenciar claramente cómo "operan" los prejuicios en la práctica y desde allí, a través del ejercicio de autoformación, "espejar" con lo que ocurre en la cotidianeidad de otras experiencias con casos que no son "extremos" pero donde j es posible reconocer el peso de estas "discriminaciones y prejuicios" que parecen banales pero que tienen efectos en la construcción de relaciones dentro de cada espacio. En todo caso, el aprendizaje y elejercicio de autoformación, que se menciona al final de este apartado, visibilizan y ponen en discusión estas temáticas 
sensibles que resultan esquivas y no evidentes cuando se proponen dinámicas de discusión en los colectivos.

b) Este proceso debe ir ajustándose (y modificando) a medida que se desarrolla: el proceso es distinto para cada compañero/a que se incorpora, así como de los contextos relacionales que lo reciben, y no siempre las herramientas pensadas son correctas 0 suficientes. Una buena predisposición por parte de los compañeros para superar las dificultades y las diferencias iniciales y crear un contexto en que se pueda sentir cómodo resulta fundamental y, como señala el punto anterior, esto debe ser planificado. De hecho, en CTP se intentó varias veces, no siempre con éxito, colaborar con proyectos como Envión o el Patronato de los Liberados para sumar nuevos socios. Estas experiencias "sin éxito", sin embargo, generaron aprendizajes que posibilitaron que, con Christian, un pibe que venía del Programa Envión y protagonista de esto, el sentido profundo de esta "excepción" lograra finalmente ser incorporado y compartido por el resto de los asociados. El proceso fue progresivo por ambos lados. Por una parte, fue fundamental la voluntad de hacerse cargo de responsabilidades y las ganas de aprender de Christian acompañado por los profesionales y colaboradores del Programa; y por otra, fueron claves el trabajo institucional, la buena voluntad por parte de los compañeros de abrir un espacio de relación, también personal, para que pudiera sentirse parte.

En términos del ejercicio de autoformación para este aprendizaje, en la Plataforma propusimos como recurso trabajar sobre base de ejemplos ficcionalizados de "Fichas de solicitud de incorporación"9 como asociado que se pueden descargar de ella junto con la Guía de Implementación.10 La idea es promover la discusión en grupos acerca de los motivos por los que aceptarían o no la incorporación de cada perfil. El ejercicio sobre el ejemplo ficcionalizado busca poner en discusión criterios reales existentes entre cada uno de nosotros que expresan prejuicios no evidentes y, en consecuencia, potenciales formas de discriminación sobre un/a futuro/a compañero/a. La clave está en que nunca los ejemplos son unívocos ni ideales, es decir, son ambiguos y contradictorios, y eso hace emerger las tensiones que se apoyan en prejuicios sobre las cualidades que deberían tener las/os compañeros que se proponen para ingresar.

\section{Reflexiones finales}

A modo de conclusión, quisiéramos destacar tres cuestiones que a nuestro entender constituyen aportes de este proyecto colaborativo con la intención de que puedan contribuir a futuras experiencias de extensión.

La primera, relativa a la relación entre cultura y territorio en los términos en que la hemos definido anteriormente. En este punto, nos interesa señalar dos desplazamientos. Uno respecto de pensar que las experiencias de gestión colectiva del trabajo resultan productivas culturalmente solo en cuanto a "abrirse" a la comunidad, integrando a otros actores y prác-

9) Se puede acceder al material desde el siguiente enlace: http://colabor.com.ar/wp-content/uploads/2017/08/ fichas.pdf

10) Se puede acceder al material desde el siguiente enlace: http://colabor.com.ar/2017/08/30/trabajar-con-nuestros-prejuicios/ 
ticas "no productivos" localizados "en el territorio" a través de experiencias como bachilleratos populares, bibliotecas, centros culturales, centros de salud, etc. Desde luego, estas acciones son sustantivas no solo para definir el alcance del proyecto político de estas experiencias, sino para mejorar significativamente las condiciones de vida de las poblaciones locales. No obstante, creemos que en estas experiencias también se elaboran repertorios culturales centrados específicamente en el quehacer productivo. Así, un segundo desplazamiento se sostiene en la rica experiencia acumulada en torno a las iniciativas de gestión colectiva del trabajo en nuestro país, que aportan a la elaboración de nuevas prácticas y sentidos acerca de aquello que llamamos "trabajo", "producción", "eficacia", "sustentabilidad", "democracia" y, como vimos en este artículo, "cooperativismo", recordándonos que la producción cultural de estos sentidos es en sí misma un campo en disputa. De este modo, producir y disputar el territorio también supone interpelar estos sentidos naturalizados, cristalizados en una lógica economicista que nos cuesta reconocer internalizada aun entre quienes nos pensamos por fuera de sus dominios.

La segunda cuestión que nos interesa señalar refiere a la práctica de extensión universitaria y el aporte del enfoque colaborativo en esta dirección. Como señalamos en el primer apartado, según la perspectiva colaborativa que adoptamos en el proyecto que dio origen a la Plataforma, la idea de codiseño fue un principio que organizó la dinámica de trabajo desde su inicio. Sin embargo, nos interesa señalar que esta dinámica solo puede ser sopesada como parte de una temporalidad más profunda construida con las cooperativas que participaron del proyecto en espacios variados de reflexión y debate en los que nos íbamos encontrando e identificando la necesidad de trabajar juntos y al sistematizar los aprendizajes desarrollados desde y en la práctica cotidiana. Fue a través de estas instancias periódicas, aunque discontinuas, que tuvo lugar, principalmente en los Encuentros de Economía de los/as Trabajadores/as, que la idea de valorizar el aporte pedagógico y no meramente vivencial y descriptivo de estas prácticas podía tener lugar. Por un lado, esto implicó salir de los abordajes curriculares y el desarrollo secuencial de los procesos de aprendizaje, obligándonos a lidiar con dinámicas más sinuosas y recursivas. Por otro, nos enfrentamos al desafío de contornear un relato épico, "color de rosa", que suele primar al plasmar en formato audiovisual experiencias de este tipo. El enfoque colaborativo fue así movilizado en pos de construir herramientas de formación autogestiva, valorando saberes y aprendizajes desde la experiencia colectiva donde se destaca el carácter interactivo, relacional, intersubjetivo y vívido de su proceso de producción, de manera de que pudieran ser replicados en otras experiencias y en otros territorios. Lejos de habilitar esta transferencia, el relato "color de rosa" no hace más que socavarla en la medida en que impide reconocernos en los errores, las dificultades, las contramarchas, las tensiones, los malestares, las incomodidades, los fracasos que son parte sustantiva de procesos de lucha y organización colectiva que hacen carne en estas experiencias. Más que un "hacer compartido", el enfoque colaborativo supuso someter este proceso a tensiones y perspectivas cruzadas donde, como insiders y outsiders cambiábamos frecuentemente de roles, permitiendo que a partir de la confrontación y el contraste recuperáramos puntos críticos de gran productividad para ampliar los alcances de los aprendizajes a otras experiencias de gestión colectiva del trabajo, más allá de las iniciativas particulares por las que habían sido elaborados inicialmente. 
Por último, una tercera cuestión refiere a los propios códigos y soportes de la producción de conocimiento colaborativo en torno a la PC, en tanto nos parecía que plasmar este proceso colaborativo en los formatos del registro escrito (como libros, cuadernillos y/o manuales) suponía establecer una barrera a priori que lo alejaría de la experiencia de compañeras/os que no estaban habituados al texto escrito y que, por el contrario, manejaban con gran asiduidad formatos y contenidos audiovisuales. En tal sentido, como parte del equipo coordinador, aprendimos sobre la marcha a corrernos de los formatos prestablecidos y animarnos a explorar interfaces novedosas, desde una perspectiva pedagógica y no expositiva. En el camino fuimos encontrando sinergias entre el enfoque colaborativo y el registro audiovisual que favorecían el involucramiento de nuestras/os compañeras/os en debates y reflexiones, de modo mucho más vívido que cuando proponíamos trabajar sobre un escrito. Esta experiencia, creemos, puede ser un aporte de peso a la hora de diseñar dispositivos de extensión universitaria crítica y reflexiva desde el territorio, en los términos a los que hicimos referencia al inicio de este artículo.

En síntesis, desde esta experiencia buscamos aportar a la valorización de la potencia instituyente y transformadora de estas prácticas de gestión colectiva del trabajo y a una búsqueda colectiva de fortalecimiento de la dimensión productiva y reproductiva cooperativa como forma posible y constantemente abierta de organizar lo común y de articular y aprender juntos entre diferentes subjetividades. Como la elaboración misma de esta Plataforma, diseñada y pensada como un proceso en experimentación, un espacio de reflexión abierto que esperamos siga alimentándose de de más y nuevos aprendizajes.

\section{Referencias bibliográficas}

Altschuler, B. (2013). Territorio y desarrollo: aportes de la geografía y otras disciplinas para repensarlos. Theomai, (27-28), 64-79

Arzeno, M. (2018). Extensión en el territorio y territorio en la extensión. Aportes a la discusión desde el campo de la Geografía. +E: Revista De Extensión Universitaria, 8(8), 3-11. Santa Fe, Argentina: Ediciones UNL. doi:10.14409/extension.v8i8.Ene-Jun.7709

Baraldo, N. (2010). Educación y Movimientos sociales contemporáneos: ¿qué prácticas? ¿qué categorías América Latina en el nuevo milenio: procesos, crisis y perspectivas. En Oliva, E.; Peirano, A.; Prudant, E. y Ruiz, J. (Eds.). América Latina en el nuevo milenio: procesos, crisis y perspectivas (pp. 255-276). Santiago de Chile: Centro de Estudios Culturales Latinoamericanos Facultad de Filosofía y Humanidades - Universidad de Chile. Carenzo, S. y Fernández Alvarez, M.I. (2017). Défis pour une ethnographie collaborative des secteurs populaires: vendeurs ambulants et ramasseurs de déchets à Buenos Aires". Ponencia presentada en el Seminario del Laboratoire d'ethnologie et de sociologie comparative (LESC), Université Paris Nanterre, Paris, 4 junio.

Elizalde, R. (2010). Movimientos sociales y estrategias socioeducactivas: experiencias en empresas recuperadas y organizaciones sociales, En Neusa, M. (Org.). Trabalho associado, economia solidária e mudança social na América Latina (pp. 97-118). São Paulo: Associação das Universidades Grupo Montevidéu -PROCOAS.

Fernández Alvarez, M. I. (2015). Contribuciones antropológicas al estudio de las cooperativas de trabajo en la Argentina reciente. Revista del Centro de Estudios de Sociología del Trabajo, 7, 37-63.

— (2016) La potencialidad de las situaciones truncas para el estudio de la política colectiva. En Fernández Álvarez, M.I. (Ed.). Hacer juntos(as). Dinámicas, contornos y relieves de la política colectiva (pp. 223-244). Buenos Aires: Biblos. 
Fernández Álvarez, M.I. y Carenzo, S. (2012). Ellos son los compañeros del CONICET: el vínculo con organizaciones sociales como desafío etnográfico. PUBLICAR - En Antropología y Ciencias Sociales, X(12), 9-34.

Rincón Gamba, L. (2012). Territorios y sujetos de la economía social y solidaria. Otra Economía, 6(10), 24-36. Gago, V. (2017). Intelectuales, experiencia y investigación militante. Nueva Sociedad, (268), 65-76.

Guelman, A. (2012). Formación para el trabajo en una empresa recuperada: Trabajo territorial y formación política. //CE, 3(1), 69-82.

Gutiérrez Aguilar, R. (2015). Horizonte comunitario-popular. Antagonismo y producción de lo común en América Latina, Puebla: ICSY-BUAP.

Haesbaert, R. (2013). Del mito de la desterritorialización a la multiterritorialidad. Cultura y representaciones sociales, 8(15), 9-42.

Hale, C. (2006). Activist Research vs. Cultural Critique: Indigenous Land Rights and the Contradictions of Politically Engaged Anthropology. Cultural Anthropology, 21(1), 96-120.

Hintze, S. (2007). Políticas sociales argentinas en el cambio de siglo. Conjeturas sobre lo posible. Buenos Aires: Espacio Editorial.

Jimeno, M. (2008). La vocación crítica de la antropología en Latinoamérica. Antípoda. Revista de Antropología y Arqueología, (1), 43-65

Leyva Solano, X. y Speed, S. (2008). Hacia la investigación descolonizada: nuestra experiencia de co-labor. En Leyva, X.; Burguete, A.; Speed, S. (Coords.). Gobernar (en) la diversidad: experiencias indígenas desde América Latina. Hacia la investigación de co-labor (pp. 15-38) México: CIESAS-FLACSO.

Massey, D. (2012). Un sentido global del lugar. Barcelona: Icaria Espacios Críticos.

Michi, N.; Vila, D. y Di Matteo, A.J. (2014). Docencia, extensión y producción de conocimientos. Una experiencia para sumarnos a los debates sobre curricularización. Ponencia presentada en el VI Congreso Nacional de Extensión Universitaria, Universidad Nacional de Rosario, 16 al 19 septiembre.

Nonaka, I. y Takeuchi, H. (1995). The knowledge-creating company: How Japanese Companies Create the Dynamics of Innovation. New York: Oxford University Press.

Polanyi, M. (1983) [1966]. The Tacit Dimension. Gloucester: Peter Smith.

Rappaport, J. (2007). Más allá de la escritura: la epistemología de la etnografía en colaboración. Revista Colombiana de Antropología, (43), 197-229.

Reygadas, L. (2002). Producción simbólica y producción material: metáforas y conceptos en torno a la cultura del trabajo. Nueva Antropología, XVIII(60), 101-119.

Scott, J.C. (1998). Seeing like a State: How certain schemes to improve the human condition have failed. Connecticut: Yale University Press

Tommasino, H. y Stevenazzi, F. (2016). Reflexiones en torno a las prácticas integrales en la Universidad de la República. +E: Revista De Extensión Universitaria, 6(6), 120-129. Santa Fe, Argentina: Ediciones UNL. doi: 10.14409/extension.v1i6.6320

Tommasino, H. y A. Cano. (2016). Modelos de extensión universitaria en las universidades latinoamericanas en el siglo XXI: tendencias y controversias. Universidades, (67), 7-24.

Vuotto, M. (2007). El cooperativismo de trabajo y la promoción del empleo. En Vuotto, M. (Comp.). Modelo para armar La co-construcción de políticas públicas en el campo de la economía social (pp. 137-161). Buenos Aires: Prometeo.

Wegner, E. (2001). Comunidades de práctica: aprendizaje, significado e identidad. Madrid: Paidós. 